\title{
Similarity of intermittency characteristics of temperature and transverse velocity
}

\author{
G. $\mathrm{Xu}^{*}$ \\ School of Civil Engineering, University of Sydney, NSW 2006, Australia
}

T. Zhou $^{\dagger}$

School of Mechanical and Aerospace Engineering, Nanyang Technological University, Singapore 639798

S. Rajagopalan

Discipline of Mechanical Engineering, University of Newcastle, NSW 2308, Australia

(Received 11 April 2007; revised manuscript received 26 July 2007; published 2 October 2007)

\begin{abstract}
The intermittency characteristics of the longitudinal $(u)$ and transverse $(v)$ velocity and temperature $(\theta)$ at similar Taylor microscale Reynolds numbers have been compared in a turbulent round jet. We examine the scaling exponents $\zeta_{\alpha}(n)(\alpha=u, v$, and $\theta)$ of structure functions $\langle(\delta \alpha)\rangle^{n}$, the intermittency exponents $\mu_{\alpha}$ based on individual and mixed sixth-order structure functions, the scaling exponents $\tau^{\alpha}(n)$ of the locally averaged energy and temperature dissipation rates approximated by $(\partial \alpha / \partial x)^{2}$, the flatness factors of the derivatives $\partial \alpha / \partial x$, and the probability density functions (PDFs) of $\partial \alpha / \partial x$, the increment $\delta \alpha$ and $(\partial \alpha / \partial x)^{2}$. It is found that $v$ and $\theta$ are similar in terms of their intermittency characteristics. They are more intermittent than $u$. The scaling exponent $\zeta_{v}(n)$ is marginally larger than $\zeta_{\theta}(n)$. The intermittency exponent $\mu_{\theta}$ is smaller than $\mu_{v}$ based on the estimate of mixed sixth-order structure functions, while $\mu_{\theta}$ is nearly equal to $\mu_{v}$ based on the estimate of individual sixth-order structure functions. The temperature dissipation rate is more intermittent than the turbulent energy dissipation rate, as indicated by $\tau^{\alpha}(n)$. The flatness factor of $\partial \theta / \partial x$ is marginally larger than that of $\partial v / \partial x$. The PDFs of $\partial \theta / \partial x, \delta \theta$, and $(\partial \theta / \partial x)^{2}$ show the strongest departure from the Gaussian distribution.
\end{abstract}

DOI: 10.1103/PhysRevE.76.046302

PACS number(s): 47.27.wg

\section{INTRODUCTION}

It is now well established that, over a scaling range (SR), moments of $\delta \alpha[\equiv \alpha(x+r)-\alpha(x)$, where $\alpha=u$ (longitudinal velocity fluctuation), $v$ (transverse velocity fluctuation), and $\theta$ (temperature fluctuation) and $r$ is the separation between the two points in the longitudinal direction] exhibit an anomalous behavior in that they deviate from the Kolmogorov [1] [K41] or K41-Obukhov [2] [K41-O49] scaling (e.g., [3-10]). Hereafter, we use SR rather than inertial range (IR) since an IR can be identified unambiguously only at infinite Reynolds numbers. In the IR, the Kolmogorov equation reduces to the "4/5" law. For flows of finite Reynolds numbers, approach to the law is gradual. Assume that the moments of $|\delta u|,|\delta v|$, and $|\delta \theta|$ in the SR are represented by power laws, viz.,

$$
\left\langle|\delta \alpha|^{n}\right\rangle \propto r^{\zeta \alpha}(n),
$$

where angular brackets denote time averaging and $\zeta_{\alpha}(n)$ are the scaling exponents of $\left\langle|\delta \alpha|^{n}\right\rangle$. The departure of $\zeta_{\alpha}(n)$ from K41 and K41-O49 predictions increases as the order of the structure functions increases (e.g., $[3,6,11,12]$ ) and is larger for $\left\langle|\delta \theta|^{n}\right\rangle$ and $\left\langle|\delta v|^{n}\right\rangle$ than for $\left\langle|\delta u|^{n}\right\rangle$, at least for nonhomogeneous turbulent shear flows. This suggests that $v$ and $\theta$ may be more intermittent than $u$. Sreenivasan [13] pointed

\footnotetext{
*Corresponding author; ggxu@optusnet.com.au

†Present address: School of Civil and Resource Engineering, The University of Western Australia, 35 Stirling Highway, Crawley WA 6009, Australia.
}

out that the similarity between the slopes of the temperature spectrum and that of the transverse velocity component is not coincidental and suggested that, in nonhomogeneous shear flows, "the scalar field attains a semblance of universality only if the velocity field in its entirety is universal." It has been found that the scaling exponents $\zeta_{\alpha}(n)$ inferred from the transverse velocity and temperature increments are nearly equal (e.g., [14]). A question which arises is whether the intermittency characteristics of $v$ are more similar to $\theta$ than to those of $u$. The main aim of this study is to compare the intermittency characteristics of $v$ and $\theta$, as determined by various methods. In particular, we examine the intermittency exponents $\mu_{v}$ and $\mu_{\theta}$, the flatness factors of the derivatives $\partial v / \partial x$ and $\partial \theta / \partial x$, the probability density functions (PDF's) of $\partial v / \partial x, \partial \theta / \partial x, \delta v, \delta \theta,(\partial v / \partial x)^{2}$, and $(\partial \theta / \partial x)^{2}$, and the scaling exponents of the locally averaged energy and temperature dissipation rates $\varepsilon_{\alpha}$, which are approximated by the following relations by assuming isotropy:

$$
\begin{aligned}
& \varepsilon_{u}=15 \nu(\partial u / \partial x)^{2}, \\
& \varepsilon_{v}=7.5 \nu(\partial v / \partial x)^{2}, \\
& \varepsilon_{\theta}=3 \alpha_{1}(\partial \theta / \partial x)^{2},
\end{aligned}
$$

where $\nu$ is the kinematic viscosity of the fluid and $\alpha_{1}$ is the thermal diffusivity of the fluid.

\section{EXPERIMENTAL CONDITIONS}

The fluctuations $u, v$, and $\theta$ were measured on the axis of a turbulent round jet. The jet was supplied by a variable 
centrifugal blower through an axisymmetric nozzle with a 10:1 contraction ratio. The measurements were carried out at $x / d=40$ (the jet nozzle diameter $d=55 \mathrm{~mm}$ ), where the flow is approximately self-preserving; this was inferred from the distributions of Reynolds normal and shear stresses. An $X$ wire was used to measure the fluctuations $u$ and $v$ for the longitudinal Taylor microscale Reynolds numbers $R_{\lambda}$ $=235-495$ without heating the jet [15]. The spanwise separation between the two wires of the $X$ probe was $1 \mathrm{~mm}$. The sensing elements were made of Pt-10\% Rh Wollaston wire with a diameter of $2.54 \mu \mathrm{m}$ and approximately $0.51 \mathrm{~mm}$ long. The fluctuations $u$ and $\theta$ were measured simultaneously for $R_{\lambda}=385-550[9,16]$. The air was heated by an electrical fan heater located at the blower entrance. The complete tunnel was insulated (25-mm-thick insulating foam with a metallic foil overlay) so as to obtain a uniform and symmetrical (with respect to the jet axis) mean temperature profile at the nozzle exit plane. The mean temperature was uniform to within $\pm 1 \%$ at the nozzle exit plane. The fluctuations $u$ and $\theta$ were measured with a hot wire (Pt-10\% Rh) of $1.27 \mu \mathrm{m}$ in diameter and $0.26 \mathrm{~mm}$ in length, and a cold wire (Pt-10\% $\mathrm{Rh})$ of $0.63 \mu \mathrm{m}$ in diameter and $0.6 \mathrm{~mm}$ in length. The wires were parallel to each other and separated in the radial direction by $0.5 \mathrm{~mm}$. The hot wire was operated in a constant temperature mode at an overheat ratio of 1.5 . The cold wire was operated in a constant current $(0.1 \mathrm{~mA})$ mode to measure the temperature fluctuation $\theta$. Velocity and temperature signals were digitized on a PC using a 12 bit A/D converter at a sampling frequency equal to two times cutoff frequency determined after examining the spectrum of the time derivative of $u, v$, and $\theta$ on a two-channel spectrum analyzer (HP3582A); electronic noise first become noticeable at this frequency. The signals were subsequently transferred to a personal computer for further analysis.

\section{RESULTS AND DISCUSSION}

The K41 scaling is based on the isotropic assumption. Local isotropy is checked by using the following relation between the second-order structure functions of the longitudinal and transverse velocity fluctuations (e.g., [17])

$$
\left\langle(\delta v)^{2}\right\rangle=\left(1+\frac{r}{2} \frac{\partial}{\partial r}\right)\left\langle(\delta u)^{2}\right\rangle .
$$

The calculated $\left\langle\left(\delta v^{*}\right)^{2}\right\rangle$ based on Eq. (5) is shown in Fig. 1, together with the measured $\left\langle\left(\delta v^{*}\right)^{2}\right\rangle$, for $R_{\lambda}=495$. An asterisk denotes normalization by the Kolmogorov length scale $\eta$ $\left[\equiv\left(\nu^{3} /\left\langle\varepsilon_{u}\right\rangle\right)^{1 / 4}\right]$ and velocity scale $U_{K}(\equiv \nu / \eta)$. The figure indicates that isotropy is satisfied reasonably, particularly in the scaling range (SR), which was determined from the distribution of $r^{*-2 / 3}\left\langle\left(\delta u^{*}\right)^{2}\right\rangle$ and $r^{*-1}\left\langle\left(\delta u^{*}\right)^{3}\right\rangle$ to be within the range $26 \leqslant r^{*} \leqslant 253[9,12]$. It was demonstrated that local isotropy is dependent on Reynolds numbers (e.g., $[18,19])$. For laboratory lows, the effect of finite Reynolds numbers on local isotropy is not possible to exclude. In a review on anisotropy in turbulent flows, Biferale and Procaccia [[20], and references therein] suggested a method of $\mathrm{SO}(3)$ decomposition to disentangle the effect of anisotropy on the scaling

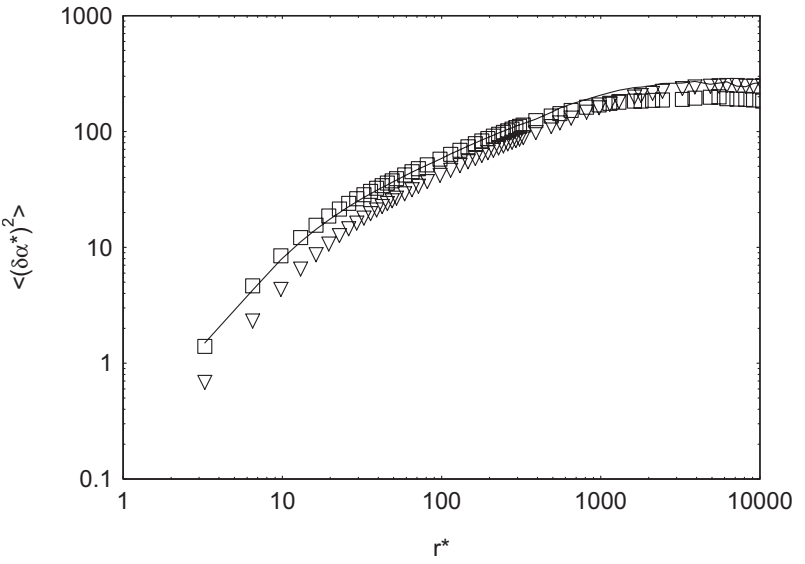

FIG. 1. The second-order structure functions of $u$ and $v$ at $R_{\lambda}$ $=495$. $\nabla, u$ measured; $\square, v$ measured; line, $v$ calculated based on Eq. (5).

exponents of structure functions. Although the method is promising and encouraging results have been obtained, the exact projection via spherical harmonics on each separate sector is not straightforward even for numerical simulation data (e.g., [21]). The SO(3) decomposition has not been used to disentangle the effect of anisotropy since the degree of anisotropy is small, the effect of finite Reynolds numbers is not possible to exclude for laboratory flows, and incomplete information on the velocity field is available in the present experiments.

First we examine the scaling exponents $\zeta_{\alpha}(n)$. Estimates of $\zeta_{u}(n), \zeta_{v}(n)$, and $\zeta_{\theta}(n)$ were determined from least-squares linear regressions to $\ln \left\langle|\delta u|^{n}\right\rangle, \ln \left\langle|\delta v|^{n}\right\rangle$, and $\ln \left\langle|\delta \theta|^{n}\right\rangle$ vs $\ln (r)$ in the SR. This is usually referred to as the direct method [3]; for each regression, the squared correlation coefficient was greater than 0.996. The extended self-similarity (ESS) method [4], i.e., plotting $\ln \left\langle|\delta u|^{n}\right\rangle, \ln \left\langle|\delta v|^{n}\right\rangle$, and $\ln \left\langle|\delta \theta|^{n}\right\rangle$ against $\ln \left\langle|\delta u|^{3}\right\rangle$, was also used. The exponents estimated by the ESS method are about 3\% smaller than those estimated by the direct method. The scaling exponents $\zeta_{u}(n), \zeta_{v}(n)$, and $\zeta_{\theta}(n)$, obtained using the direct method, are shown in Fig. 2

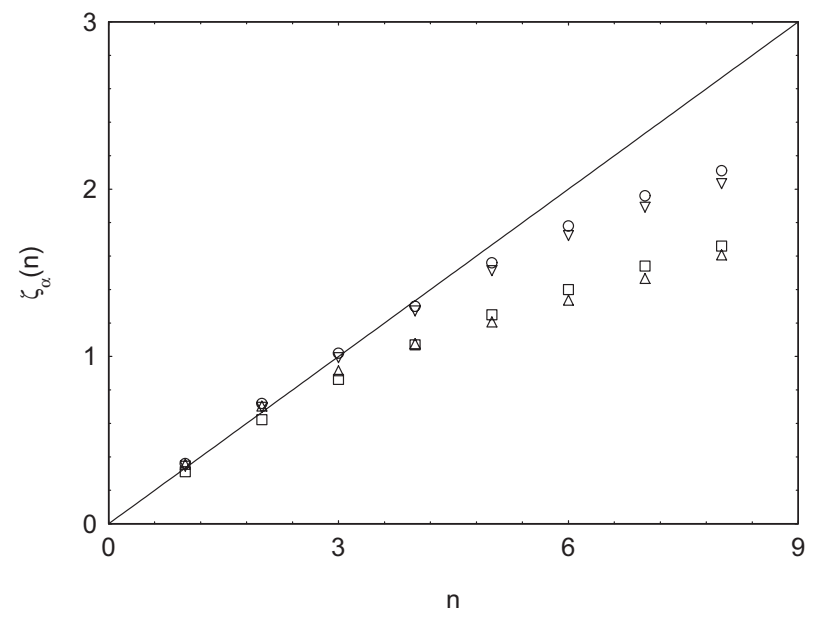

FIG. 2. Scaling exponents $\zeta_{\alpha}(n)$ for $R_{\lambda}=495(u$ and $v)$ and 550 $(u$ and $\theta)$. $\bigcirc, R_{\lambda}=550, \alpha=u ; \triangle, 550, \theta ; \nabla, 495, u ; \square, 495, v$. 
for two values of $R_{\lambda}$ (495 and 550). The uncertainty for the scaling exponents was estimated to be in the range \pm 0.01 for $n=1$ to \pm 0.06 for $n=8$. Also included in the figure is the K41-O49 prediction $\left[\zeta_{\alpha}(n)=n / 3\right.$ with $\alpha=u, v$, or $\left.\theta\right]$. The exponents $\zeta_{u}(n), \zeta_{v}(n)$, and $\zeta_{\theta}(n)$ depart from this prediction. The departure increases with increasing $n . \zeta_{v}(n)$ is marginally larger than $\zeta_{\theta}(n)$. Both $\zeta_{\theta}(n)$ and $\zeta_{v}(n)$ are significantly smaller than $\zeta_{u}(n)$, suggesting that $\theta$ and $v$ are more intermittent than $u$.

The $n$ th-order structure functions of velocity are given by (e.g., [22])

$$
\left\langle(\delta u)^{n}\right\rangle=C_{n} r^{n / 3}\left\langle\varepsilon_{r}^{n / 3}\right\rangle,
$$

where $C_{n}$ are universal constants which depend on the particular values of $n$.

The sixth-order structure function is

$$
\left\langle(\delta u)^{6}\right\rangle=C_{6} r^{2}\left\langle\varepsilon_{r}^{2}\right\rangle .
$$

Therefore, the scaling exponent of $\left\langle(\delta u)^{6}\right\rangle$ is

$$
\zeta(6)=2-\mu,
$$

where $\mu$ is the scaling exponent of the energy dissipation rate $\left\langle\varepsilon_{r}^{2}\right\rangle$.

Dimensional arguments presented by Antonia and Van Atta [23] suggested that for a Prandtl number $\operatorname{Pr} \approx 1$, the mixed structure functions of the velocity and temperature $\theta$ in the SR are

$$
\left\langle(\delta u)^{n}(\delta \theta)^{m}\right\rangle=C_{n m} r^{(n+m) / 3}\left\langle\varepsilon_{r}^{n / 3-m / 6} \varepsilon_{\theta r}^{m / 2}\right\rangle,
$$

where $C_{n m}$ are universal constants which depend on the particular values of $n$ and $m$.

From Eq. (9), we can obtain the mixed sixth-order structure function of $u$ and $\theta$,

$$
\left\langle(\delta u)^{2}(\delta \theta)^{4}\right\rangle=C_{24} r^{2}\left\langle\varepsilon_{\theta r}^{2}\right\rangle .
$$

As a result, the scaling exponent of $\left\langle(\delta u)^{2}(\delta \theta)^{4}\right\rangle$ is

$$
\zeta_{u} \theta(2,4)=2-\mu_{\theta},
$$

where $\mu_{\theta}$ is the scaling exponent of the temperature dissipation rate $\left\langle\varepsilon_{\theta r}^{2}\right\rangle$.

For comparison purposes and similarity of intermittency characteristic of $v$ and $\theta$, it is assumed that Eqs. (8) and (11) are also applicable to $v$ and $\theta$, viz.,

$$
\begin{gathered}
\mu_{\alpha}=2-\zeta_{\alpha}(6), \\
\mu_{\alpha}=2-\zeta_{u \alpha}(2,4) .
\end{gathered}
$$

The intermittency exponents $\mu_{\alpha}$ can be estimated by using Eqs. (8a) and (11a). We found that $\mu_{\theta}=0.17$ and $\mu_{v}=0.43$ based on Eq. (8a). On the other hand, when Eq. (11a) is used, $\mu_{\theta}(=0.67)$ is nearly equal to $\mu_{v}(=0.6)$; their magnitudes are significantly larger than that of $\mu_{u}(=0.20)$. The two intermittency exponent definitions, i.e., Eqs. (8a) and (11a), result in different estimates for $\mu_{v}$ and $\mu_{\theta}$. Xu [16] found that different methods yield different values of $\mu_{\alpha}$ and the difference also depends on the choice of the SR. The similarity between the scaling of the mean turbulent energy $\left\langle q^{2}\right\rangle\left(\equiv\left\langle u^{2}\right\rangle+\left\langle v^{2}\right\rangle\right.$

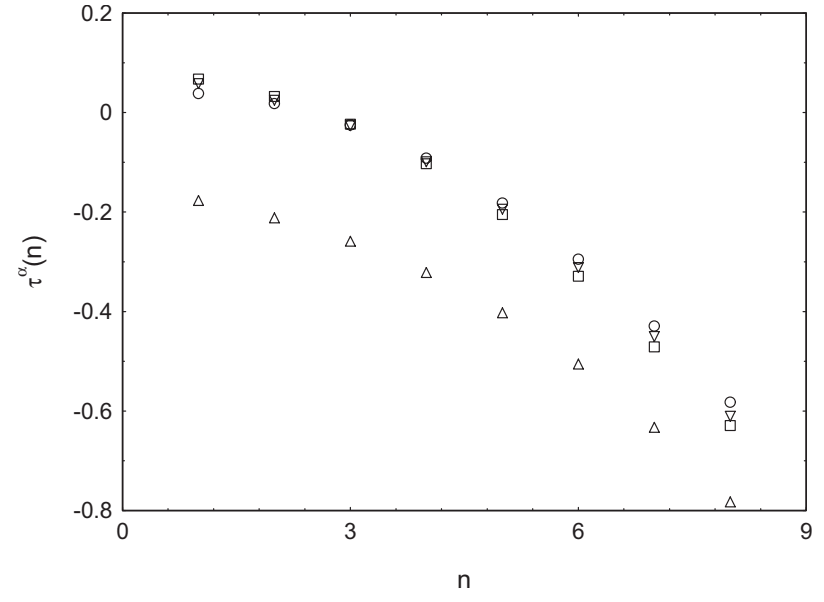

FIG. 3. Scaling exponents $\tau^{\alpha}(n)$. Symbols are as in Fig. 2.

$+\left\langle w^{2}\right\rangle$, where $w$ is the spanwise velocity fluctuation) and $\langle\theta\rangle^{2}$ was studied by Antonia and Smalley [24] in a slightly heated rough wall boundary layer. They found that the intermittency exponent of $v$ based on Eq. (8a) is larger than that of $\theta$ (their Fig. 11). This is in agreement with the present results. However, their magnitudes of $\mu_{\alpha}$ are significantly larger than those for the present jet. This difference may be ascribed to the strong shear and/or anisotropy in the rough wall boundary layer of Antonia and Smalley [24] while the present data are obtained on the jet centerline without shear.

Within the frame of the refined similarity hypothesis [22], the scaling of the $\alpha$ structure functions is

$$
\zeta_{\alpha}(n)=n / 3+\tau^{\alpha}(n / 3),
$$

where $\tau^{\alpha}(n / 3)$ are the scaling exponents of the locally averaged energy and temperature dissipation rates. They are inferred from the distributions of $\ln \left\langle\varepsilon_{\alpha r}^{n / 3}\right\rangle$ vs $\ln (r)$ over the SR, i.e.,

$$
\left\langle\left(\varepsilon_{\alpha r}\right)^{n}\right\rangle \propto r^{\tau^{\alpha}(n)} .
$$

Hereafter, $\partial \alpha / \partial x$ are obtained from the $\alpha$ signals measured by using Taylor's hypothesis. It is found that the magnitude of $\tau^{\theta}(n)$ is much smaller than that of $\tau^{v}(n)$ (Fig. 3). The uncertainty for the scaling exponents in Fig. 3 was estimated to be in the range \pm 0.01 for $n=1$ to \pm 0.06 for $n=8$. This indicates that the temperature dissipation rate, represented by $(\partial \theta / \partial x)^{2}$, is more intermittent than the turbulent energy dissipation rate, represented by $(\partial v / \partial x)^{2} . \tau^{u}(n)$ and $\tau^{\nu}(n)$ are nearly equal, which indicates that within the frame of the refined similarity hypothesis [Eq. (12)], the already established inequality $\left[\zeta_{u}(n)>\zeta_{v}(n)\right]$ cannot be associated with the scaling exponents $\tau^{\alpha}(n)$ of "two" different dissipation fields represented by $(\partial u / \partial x)^{2}$ and $(\partial v / \partial x)^{2}$, respectively. This inequality is more likely to be due to different smallscale intermittencies associated with $(\partial u / \partial x)^{2}$ and $(\partial v / \partial x)^{2}$, and possibly the different way in which the large-scale anisotropy affects these "two" fields.

The intermittency can be quantified by the flatness factor. The $R_{\lambda}$ dependence of the flatness factors of $\partial \alpha / \partial x, F_{\partial \alpha / \partial x}$, is shown in Fig. 4. Relatively few data are available for the 


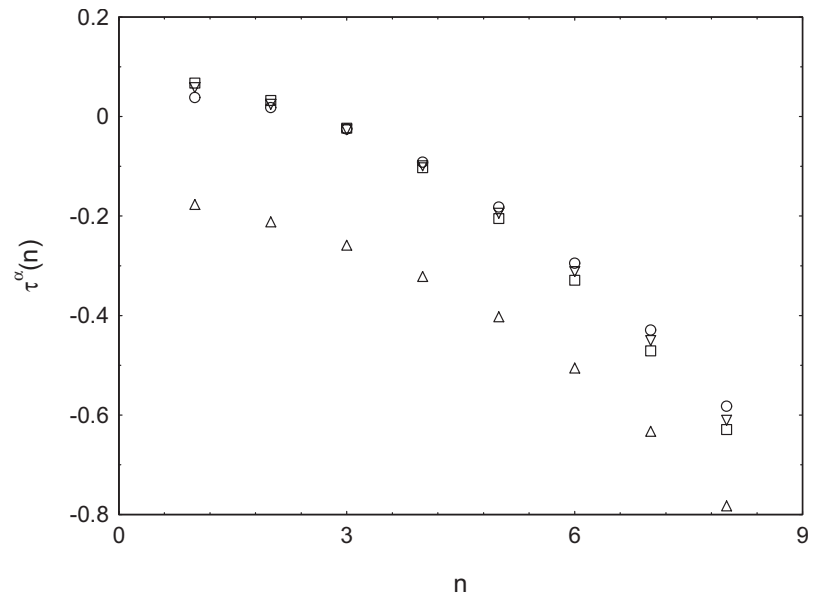

FIG. 4. Flatness factors of $\partial \alpha / \partial x$. Present jet measurements: *, $\alpha=u ; \boldsymbol{O}, v ; \boldsymbol{\square}, \theta ;-, \alpha=u[7] ; \bigcirc, v[25] ; \square, \theta[7]$.

dependence of $F_{\partial v / \partial x}$ on $R_{\lambda}$. The majority of the data for $F_{\partial v / \partial x}$ and $F_{\partial \theta / \partial x}$ in Fig. 4 come from Pearson and Antonia [25] and Sreenivasan and Antonia [7], respectively. The figure shows that $F_{\partial \theta / \partial x}$ is marginally larger in magnitude than $F_{\partial v / \partial x}$. The curve in the figure represents a least-squares fit to Sreenivasan and Antonia's [7] compilation of the flatness factor of $\partial u / \partial x$. Both $F_{\partial v / \partial x}$ and $F_{\partial \theta / \partial x}$ have a stronger $R_{\lambda}$ dependence than $F_{\partial u / \partial x}$, suggesting that $\theta$ and $v$ are more intermittent than $u$. The flatness factors of $\partial \alpha / \partial x$ are in good agreement with the $R_{\lambda}$ trend of Pearson and Antonia [25] for $F_{\partial v / \partial x}$ and Sreenivasan and Antonia [7] for $F_{\partial u / \partial x}$ and $F_{\partial \theta / \partial x}$ (Fig. 4), respectively. For isotropic turbulence, the skewness factor of $\partial u / \partial x, S_{\partial u / \partial x}$, must be negative as $-\left\langle(\partial u / \partial x)^{3}\right\rangle$ represents a production rate of mean-square turbulent vorticity (e.g., [26]). The skewness factor of $\partial \theta / \partial x, S_{\partial \theta / \partial x}$, must be zero in isotropic turbulence to satisfy the requirement of invariance with respect to reflection and rotation of the coordinate axes. The values of $S_{\partial u / \partial x}$ and $S_{\partial \theta / \partial x}$ are -0.48 and -0.60 for $R_{\lambda}=550$, respectively. The values of $S_{\partial u / \partial x}$ and the skewness factor of $\partial v / \partial x, S_{\partial v / \partial x}$, are -0.47 and 0.06 for $R_{\lambda}$

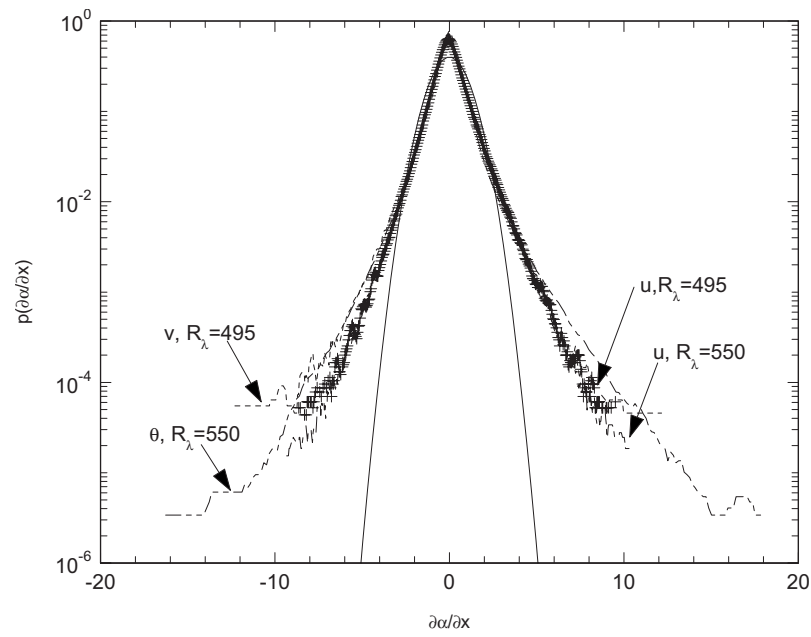

FIG. 5. PDFs of $\partial \alpha / \partial x$ for $R_{\lambda}=495(u$ and $v)$ and $550(u$ and $\theta)$. $---, R_{\lambda}=550, \alpha=u$; ---, 550, $\theta$; $+, 495, u ;---, 495, v$. Solid line: Gaussian distribution.

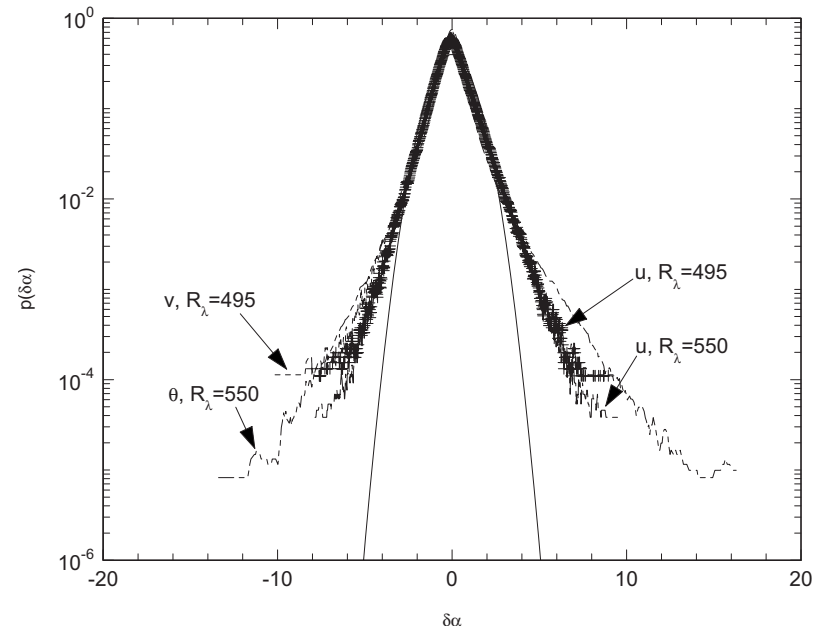

FIG. 6. PDFs of $\delta \alpha$ at $r^{*}=10$. Symbols are as in Fig. 5.

$=495$, respectively. The magnitude of $S_{\partial u / \partial x}$ agrees well with the experimental and DNS values of $0.4-0.5$ (e.g., $[18,27]$ ). $S_{\partial v / \partial x}$ is close to zero, and agrees with the measured values of Champagne [28], Tavoularis and Corrsin [29], and Mydlarski and Warhaft [18]. $S_{\partial \theta / \partial x}$ is in accord with the compilation of Sreenivasan and Antonia [7]. The nonzero value of $S_{\partial \theta / \partial x}$ might be associated with temperature ramps (e.g., [30]). This large-scale structure behavior has been observed in various turbulent shear flows.

The PDFs of the derivatives $\partial u / \partial x, \partial v / \partial x$, and $\partial \theta / \partial x$ for $R_{\lambda}=495$ and 550 are shown in Fig. 5. These distributions are closer to an exponential than a Gaussian distribution. The tails are not straight lines in this linear-logarithmic plot. Although the PDFs of $\partial u / \partial x, \partial v / \partial x$, and $\partial \theta / \partial x$ deviate significantly from the Gaussian distribution, the PDF of $\partial \theta / \partial x$ shows the strongest departure from the Gaussian distribution. The PDFs of $\delta \alpha$ are shown in Fig. 6 at $r^{*}=10$ in the dissipation range and Fig. 7 at $r^{*}=100$ in the SR. These distributions seem to display exponential tails. For the largest values of $r^{*}$, which correspond to the velocity and temperature fluctuations, the PDFs of $\alpha$ are Gaussian (results are not shown here). When $r^{*}$ increases from 0 to the largest value, the PDFs of $\delta \alpha$ change continuously from a distribution similar

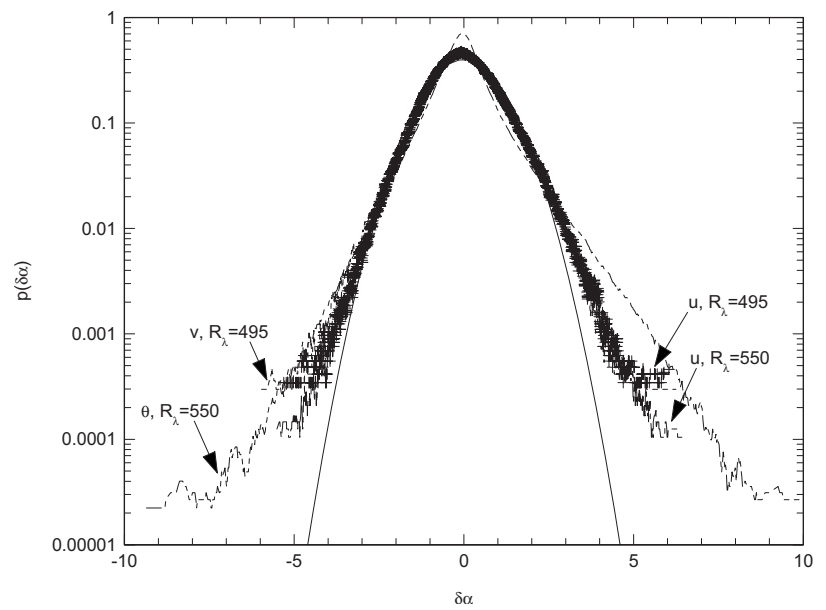

FIG. 7. PDFs of $\delta \alpha$ at $r^{*}=100$. Symbols are as in Fig. 5. 


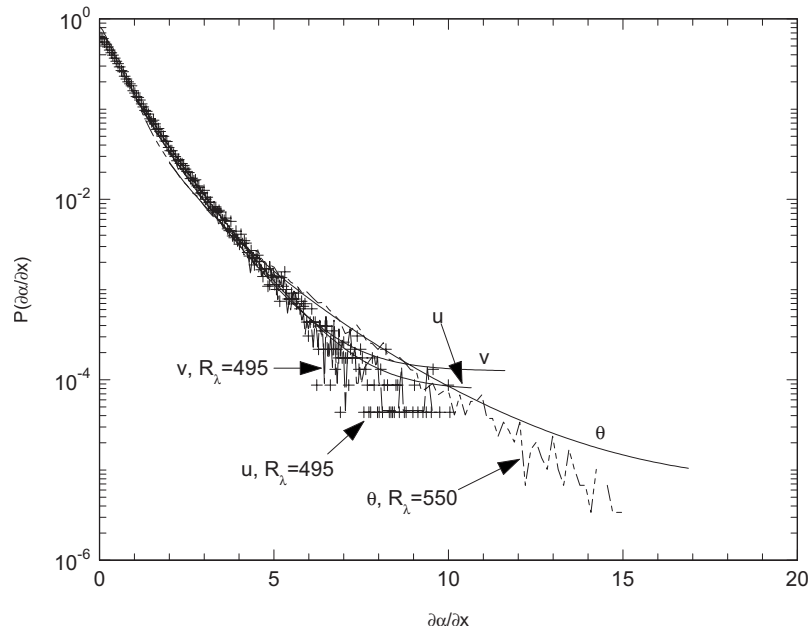

FIG. 8. Comparison of PDFs of $\partial \alpha / \partial x$ between the measurements and model [Eq. (15)]. Solid lines, by the model; dashed lines, by measurements; +, 495, $u$; ---, 495, $v$; ---, 550, $\theta$.

to those of $\partial \alpha / \partial x$ (Fig. 5) to a Gaussian distribution. For intermediate values of $r^{*}$, the tails look exponential (Fig. 7). Although the PDFs of $\delta u, \delta v$, and $\delta \theta$ deviate significantly from the Gaussian distribution, the PDF of $\delta \theta$ exhibits the largest departure, which indicates the most intermittent behavior.

Some models were suggested to describe the probability density functions (PDF's) of velocity increments $(\delta \alpha)$ and gradients $(\partial \alpha / \partial x)$ (e.g., [5,31-33]). These models are a superposition of Gaussians with different widths increasing as the separation $r$ decreases. Benzi et al. [32] used the multifractal approach and random beta model [34] to derive the PDFs of $\delta \alpha$ and $\partial \alpha / \partial x$,

$$
\begin{aligned}
P(\delta \alpha) \approx & \sum_{K=0}^{n}\left[\begin{array}{l}
n \\
K
\end{array}\right] a^{n-K}(1-a)^{K} B^{4 K / 3} l_{n}^{-1 / 3} \\
& \times \exp \left[-C B^{2 K / 3} l_{n}^{-2 / 3}(\delta \alpha)^{2}\right], \\
P\left(\frac{\partial \alpha}{\partial x}\right)= & \sum_{K=0}^{N}\left[\begin{array}{l}
N \\
K
\end{array}\right] a^{N-k}(1-a)^{k}\left[\nu /\left|\frac{\partial \alpha}{\partial x}\right|\right]^{(1+2 k) / 3} \\
& \times \exp \left[-C \nu^{(2+k) / 3}\left|\frac{\partial \alpha}{\partial x}\right|^{(4-k) / 3}\right],
\end{aligned}
$$

where $\quad C=\left(2\left\langle V_{0}^{2}\right\rangle\right)^{-1}, \quad l_{n}=2^{-n}, \quad k=K / N, \quad$ and $\quad N$ $=\ln (|\partial \alpha / \partial x| \nu) /(2 \ln 2)$. Figures 8 and 9 show comparisons between the present measurements of $P(\delta \alpha)$ at $r^{*}=100$ in the scaling range and $P(\partial \alpha / \partial x)$, and those based on the models of Eqs. (14) and (15). The models fit well the measured PDFs of the increments $\delta \alpha$ and gradients $\partial \alpha / \partial x$, but not for their tails to which only a few events contribute.

Figure 10 shows the PDFs of $(\partial \alpha / \partial x)^{2}$ for $R_{\lambda}=495$ and 550. The PDFs' tails look like a stretched exponential. These distributions are more intermittent than an exponential distribution. The PDF of $(\partial \theta / \partial x)^{2}$ shows the widest tail and the

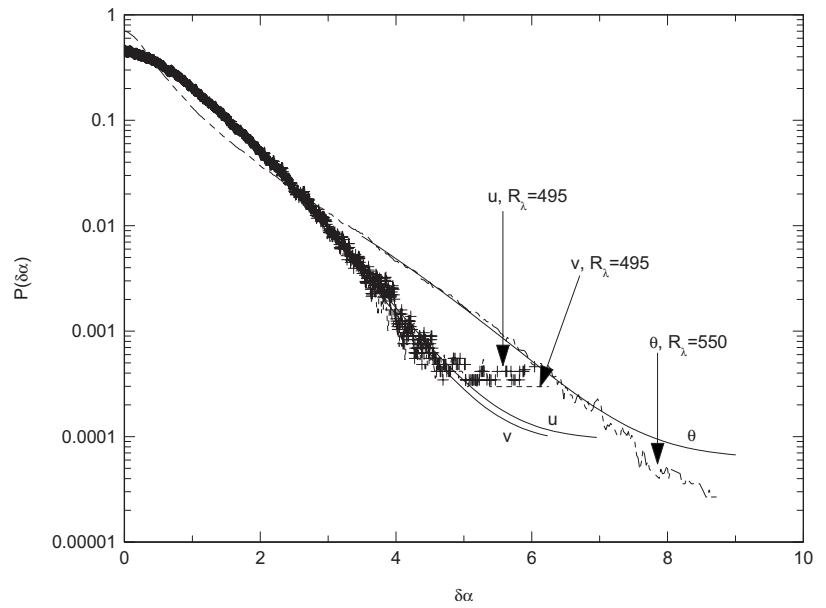

FIG. 9. Comparison of PDFs of $\delta \alpha$ between the measurements and model [Eq. (14)] at $r^{*}=100$. Solid lines, by the model; dashed lines, by measurements; - , 495, $u$; --, 495, $v$;---, 550, $\theta$.

largest departure from the Gaussian and exponential distributions, suggesting that $\theta$ is more intermittent than $u$ and $v$.

\section{CONCLUSIONS}

The intermittency characteristics of $\alpha(=u, v$, and $\theta)$ have been compared by examining the scaling exponents $\zeta_{\alpha}(n)$ for the $\alpha$ structure functions and $\tau^{\alpha}(n)$ for the local energy and temperature dissipation rates, intermittency exponents $\mu_{\alpha}$, the flatness factors of the derivatives $\partial \alpha / \partial x$, and the PDFs of $\partial \alpha / \partial x, \delta \alpha$, and $(\partial \alpha / \partial x)^{2}$. It is found that while $v$ and $\theta$ are more intermittent than $u, v$ and $\theta$ are similar in terms of their intermittency characteristics. The scaling exponent $\zeta_{v}(n)$ is marginally larger than $\zeta_{\theta}(n)$. The intermittency exponent $\mu_{\theta}=0.17$ is smaller than $\mu_{v}=0.43$ based on the estimate of mixed sixth-order structure functions $\left\langle(\delta u)^{2}(\delta \alpha)^{4}\right\rangle$, while based on the estimate of individual sixth-order structure functions $\left\langle(\delta \alpha)^{6}\right\rangle, \quad \mu_{\theta} \quad(=0.67)$ is nearly equal to

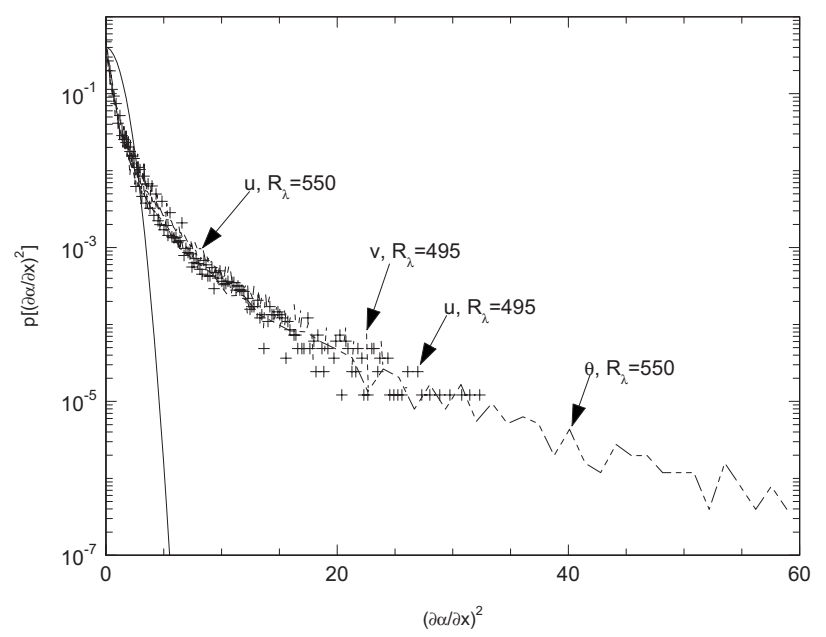

FIG. 10. PDFs of $(\partial \alpha / \partial x)^{2}$. Symbols are as in Fig. 5. 
$\mu_{v}(=0.6)$, their magnitudes being significantly larger than that $(=0.20)$ of $\mu_{u}$. The temperature dissipation rate, represented by $(\partial \theta / \partial x)^{2}$, is more intermittent than the turbulent energy dissipation rate, represented by $(\partial v / \partial x)^{2}$. The flatness factor of $\partial \theta / \partial x$ is marginally larger than that of $\partial v / \partial x$. The PDFs of $\partial \theta / \partial x, \delta \theta$, and $(\partial \theta / \partial x)^{2}$ shows the strongest departure from the Gaussian distribution, compared with those of $\partial u / \partial x$ and $\partial v / \partial x, \delta u$ and $\delta v$, and $(\partial u / \partial x)^{2}$ and $(\partial v / \partial x)^{2}$, respectively.

\section{ACKNOWLEDGMENT}

The authors would like to thank Professor R. A. Antonia for beneficial discussions.
[1] A. N. Kolmogorov, Dokl. Akad. Nauk SSSR 30, 301 (1941).

[2] A. M. Obukhov, Izv. Akad. Nauk SSSR, Ser. Geogr. Geofiz. 13, 58 (1949).

[3] F. Anselmet, Y. Gagne, E. J. Hopfinger, and R. A. Antonia, J. Fluid Mech. 140, 63 (1984).

[4] R. Benzi, S. Ciliberto, R. Tripiccione, C. Baudet, F. Massaioli, and S. Succi, Phys. Rev. E 48, R29 (1993).

[5] U. Frisch, Turbulence: The Legacy of A. N. Kolmogrov (Cambridge University Press, Cambridge, England, 1997).

[6] B. Dhruva, Y. Tsuji, and K. R. Sreenivasan, Phys. Rev. E 56, R4928 (1997).

[7] K. R. Sreenivasan and R. A. Antonia, Annu. Rev. Fluid Mech. 29, 435 (1997).

[8] W. Van de Water and J. A. Herjeiwer, J. Fluid Mech. 387, 3 (1999).

[9] G. Xu, R. A. Antonia, and S. Rajagopalan, Europhys. Lett. 49, 452 (2000).

[10] T. Gotoh, D. Fukayama, and T. Nakano, Phys. Fluids 14, 1065 (2002).

[11] R. A. Antonia, E. J. Hopfinger, Y. Gagne, and F. Anselmet, Phys. Rev. A 30, 2704 (1984).

[12] G. Xu, R. A. Antonia, and S. Rajagopalan, Europhys. Lett. 79, 44001 (2007).

[13] K. R. Sreenivasan, Phys. Fluids 8, 189 (1996).

[14] R. A. Antonia and B. R. Pearson, Europhys. Lett. 40, 123 (1997).

[15] G. Xu, R. A. Antonia, and S. Rajagopalan, Fluid Dyn. Res. 28,
311 (2001).

[16] G. Xu, Flow, Turbul. Combust. 72, 369 (2004).

[17] A. S. Monin and A. M. Yaglom, Statistical Fluid Mechanics (MIT Press, Cambridge, MA, 1975).

[18] L. Mydlarski and Z. Warhaft, J. Fluid Mech. 320, 331 (1996).

[19] G. Xu, S. Rajagopalan, and R. A. Antonia, Fluid Dyn. Res. 26, 1 (2000).

[20] L. Biferale and I. Procaccia, Phys. Rep. 43, 414 (2005).

[21] L. Biferale and M. Vergassola, Phys. Fluids 13, 2139 (2001).

[22] A. N. Kolmogorov, J. Fluid Mech. 13, 82 (1962).

[23] R. A. Antonia and C. W. Van Atta, J. Fluid Mech. 67, 273 (1975).

[24] R. A. Antonia and R. J. Smalley, Phys. Rev. E 62, 640 (2000).

[25] B. R. Pearson and R. A. Antonia, J. Fluid Mech. 444, 343 (2001).

[26] J. C. Wyngaard, J. Fluid Mech. 48, 763 (1971).

[27] R. M. Kerr, J. Fluid Mech. 153, 31 (1985).

[28] F. H. Champagne, J. Fluid Mech. 86, 67 (1978).

[29] S. Tavoularis and S. Corrsin, J. Fluid Mech. 104, 349 (1981).

[30] C. W. Van Atta, Arch. Mech. 29, 161 (1977).

[31] M. Nelkin, Phys. Rev. A 42, 7226 (1990).

[32] R. Benzi, L. Bierale, G. Paladin, and A. Vulpiani, Phys. Rev. Lett. 67, 2299 (1991).

[33] L. Chevillard, B. Castaing, E. Leveque, and A. Arneodo, Physica D 218, 77 (2006).

[34] R. Benzi, G. Paladin, G. Parisi, and A. Vulpiani, J. Phys. A 17, 3521 (1984). 